\title{
Composição química e atividade inseticida do extrato acetônico de Piper alatabaccum Trel \& Yuncker (Piperaceae) sobre Hypothenemus hampei Ferrari
}

SANTOS, M.R.A. ${ }^{1}$; LIMA, R.A. ${ }^{*}$; SILVA, A.G. ${ }^{1}$; TEIXEIRA, C.A.D. ${ }^{1}$; ALPIREZ, I.P.V. ${ }^{2}$; FACUNDO, V.A. ${ }^{2}$ ${ }^{1}$ Embrapa Rondônia - BR 364, km 5,5, Caixa Postal 127, CEP: 76815-800, Porto Velho-Rondônia, Brasil; *abo295@hotmail.com 2Laboratório de Produtos Naturais da Universidade Federal de Rondônia, BR 364, km 9,5, CEP: 78900-000, Porto Velho-Rondônia, Brasil.

\begin{abstract}
RESUMO: O presente trabalho teve como objetivo investigar a composição química e avaliar a atividade inseticida do extrato acetônico das raízes de Piper alatabaccum sobre Hypothenemus hampei. As raízes foram coletadas no campus da Universidade Federal de Rondônia, em Porto Velho-RO. Diluições de 100,0, 50,0, 25,0, 5,0 e 0,5 mg mL-1 foram utilizadas para exposição na superfície contaminada e aplicação tópica. No teste de repelência, utilizou-se as diluições de 25,0 e $100,0 \mathrm{mg} \mathrm{mL}^{-1}$. As avaliações ocorreram durante 48 horas após a exposição ao extrato. O extrato foi eficiente na mortalidade em aplicação tópica e superfície contaminada, não sendo eficiente no efeito de repelência. Estes resultados apontam para o elevado potencial inseticida do extrato de $P$. alatabaccum no controle de $H$. hampei. Identificou-se cinco compostos, sendo três amidas, dihydropiplartine piperovatina e piplartina, e duas flavona, 3, 4, $, 5,5^{\prime}, 7$ pentamethoxyflavone e 5,5',7-trimetóxi-3',4'-methylenedioxyflavone.
\end{abstract}

Palavras-chave: Coffea arabica, Piperaceae, broca-do-café, repelência.

ABSTRACT: Chemical constituents and insecticidal activity of the crude acetonic extract of Piper alatabaccum Trel \& Yuncker (Piperaceae) on Hypothenemus hampei Ferrari. The purpose of this work was to investigate the chemical composition and to evaluate the insecticidal activity of the acetonic extract of Piper alatabaccum roots on Hypothenemus hampei. Roots of $P$. alatabaccum were collected at the University of Rondônia in Porto Velho-RO. Dilutions of 100.0, 50.0, 25.0, 5.0 and $0.5 \mathrm{mg} \mathrm{mL}^{-1}$ were used for exposition in the contaminated surface and for topical application. For the repellence test, 25.0 and $100.0 \mathrm{mg} \mathrm{mL}^{-1}$ dilutions were used. The evaluations occurred during 48 hours after exposure to the extract. The extract was efficient in terms of mortality, but no repellence was observed in the bioassays. These results pointed out the high insecticidal potential of the $P$. alatabaccum extract to control $H$. hampei. Five compounds, three amids, piperovatine, dihydropiplartine and piplartine, and two flavones, 3, 4',5,5, 7-pentamethoxyflavone and 5,5',7-trimethoxy-3', 4' methylenedioxyflavone were identified.

Key words: Coffea arabica, Piperaceae, coffee berry borer, repellency.

\section{INTRODUÇÃO}

A cafeicultura é responsável por grande movimentação de recursos financeiros no Brasil, abrangendo a contratação de mão-de-obra para a realização de tratos culturais, a negociação de grandes lotes de insumos e máquinas e a comercialização do produto final (Guimarães et al., 2002).

Atualmente, o estado de Rondônia é o maior produtor de café da região Norte, respondendo por cerca de $80 \%$ da produção, e segundo maior produtor de café Robusta (Coffea canephora var. Robusta) do país, superado apenas pelo Espírito
Santo. Rondônia possui uma área plantada de 200.000 ha, cuja produção pode atingir 2 milhões de sacas beneficiadas; essa produção se concentra principalmente nas microrregiões de Cacoal, Alvorada do Oeste, Ji-Paraná e Ariquemes (Santos, 2002).

A broca-do-café (Hypothenemus hampei Ferrari) é uma praga das regiões produtoras de café, atacando frutos em qualquer estádio de maturação, podendo ocasionar perda total na produção (Barreira et al., 1990). Os altos índices de danos deste inseto em Rondônia se devem, em parte, a umidades

Recebido para publicação em 15/11/2010

Aceito para publicação em 08/11/2012

Rev. Bras. Pl. Med., Campinas, v.15, n.3, p.332-336, 2013. 
e temperaturas altas. Dependendo do nível de infestação, os prejuízos podem chegar a $21 \%$, somente pela perda de peso (Reis et al., 2002).

$\mathrm{O}$ uso de inseticidas convencionais para o controle de insetos tem sido de pouca eficiência entre os países cafeicultores da América, devido à seleção natural de linhagens de insetos resistentes e problemas ambientais, como a eliminação da fauna benéfica, a crescente contaminação do solo, água, atmosfera e seres vivos, além de danos acidentais ocasionados pela utilização. Estes fatos têm incentivado a pesquisa de métodos alternativos para o controle de insetos praga (Bustillo, 2002).

A utilização de aleloquímicos que atraem, repelem ou interrompem o processo alimentar, com a finalidade de proteger as culturas do ataque de insetos, tem sido amplamente estudada. Essas substâncias têm vantagens de serem específicas em relação às pragas, não eliminando os inimigos naturais e dificultando o processo co-evolucionário da criação de resistência pelos insetos fitófagos (Castro et al., 2005).

A família Piperaceae é composta por grande variedade de espécies, muitas vezes habitando a borda ou o estrato inferior das matas. O maior gênero desta família, Piper, inclui mais de 1.000 espécies, fazendo deste o maior gênero das angiospermas basais, as chamadas paleoervas. As espécies de Piper são distribuídas pantropicalmente e a maioria está envolvida em processos de regeneração e manutenção da diversidade das matas (Jaramillo \& Manos, 2001).

Devido à presença de grande variedade de metabólitos secundários, diversas espécies do gênero Piper têm sido avaliadas com relação ao potencial no controle de microrganismos e insetos que acometem os sistemas agrícolas. Silva et al. (2007), demonstraram a atividade inseticida dos extratos das folhas e raízes de $P$. aduncum em adultos de Aetalion sp. (Hemiptera: Aetalionidae). Fazolin et al. (2005), observaram o potencial inseticida do óleo essencial dessa mesma espécie frente a Cerotoma tingomarianus Bechyné (Coleoptera: Chrysomelidae), nas diluições de 10, 25, 50, 75, 100, 200, e $300 \mathrm{mg} \mathrm{L}^{-1}$.

Adicionalmente, Estrela et al. (2006) ao avaliar o efeito da aplicação tópica dos óleos essenciais de $P$. aduncum e $P$. hispidinervum sobre a mortalidade de Sitophilus zeamais, observaram porcentagens significativas para todas as diluições avaliadas, atingindo mortalidade superior a $70 \%$. Estes resultados, provavelmente estão relacionados ao dilapiol, componente majoritário das folhas de $P$. aduncum $(73,97 \%)$ e ao safrol componente majoritário de $P$. hispidinervum $(94,72 \%)$, uma vez que ambos já possuem atividade inseticida comprovado na literatura. Finalmente, Santos et al.,
(2010) observou o potencial inseticida do extrato acetônico das folhas de Piper hispidum Kunth sobre Hypothenemus hampei em aplicação tópica, superfície contaminada e repelência em diferentes diluições.

A espécie Piper alatabaccum Trel. e Yunck, conhecida popularmente como "João brandinho", é encontrada na Amazônia, e suas raízes são utilizadas popularmente como anestésico local, possivelmente pela presença de amidas em sua composição química (Souza \&Lorenzi, 2008). Além disso, estudos recentes mostraram a atividade larvicida dos extratos metanólicos de $P$. Tuberculatum, $P$. alatabaccum e os compostos isolados: ácido 3,4,5-trimetoxydihydrocinamic e dihydropiplartine; Piplartina, piplartina-dihydropiplartine e 5,5',7-trimetoxy-3', 4'-metilenodioxiflavone frente a $A$. darlingi (Trindade et al., 2012). Sugerindo que seus metabólitos sejam efetivos contra outras espécies de insetos, gerando alternativas de baixo custo para o controle desta praga.

Desta forma, este estudo teve como objetivo investigar a composição química e avaliar a atividade inseticida do extrato acetônico das raízes de Piper alatabaccum sobre Hypothenemus hampei.

\section{MATERIAL E MÉTODO}

As raízes de $P$. alatabaccum foram coletadas no campus da Universidade Federal de Rondônia, em Porto Velho-RO. A identificação botânica da espécie foi realizada no herbário do Instituto Nacional de Pesquisa da Amazônia (INPA), onde uma exsicata encontra-se depositada sob o número 211711.

As raízes devidamente secas e trituradas de $P$. alatabaccum $(0,9 \mathrm{~kg})$ foram extraídas com acetona $(3 L \times 3)$ à temperatura ambiente. O solvente foi destilado sob pressão reduzida fornecendo $21,0 \mathrm{~g}$. Parte deste material $(10,0 \mathrm{~g})$ foi adsorvido em sílica gel $(70,0 \mathrm{~g})$ e a mistura, sob a forma de pastilha, foi colocada em uma coluna cromatográfica e eluída com hexano, clorofórmio, acetato de etila e metanol. A fração clorofórmio $(6,3 \mathrm{~g})$ foi novamente submetida à cromatografia em coluna de gel de sílica e eluída com misturas de hexano e clorofórmio em polaridade crescente para o isolamento dos metabólitos secundários encontrados neste estudo.

Nos bioensaios foram utilizados insetos adultos de $H$. hampei, oriundos da criação estoque da Embrapa Rondônia, onde foram coletados em 350 frutos maduros de café, armazenados em caixas de madeira ( $26 \times 37 \times 13 \mathrm{~cm})$, acondicionadas em gaiolas com proteção de tela de filó, à temperatura de $25 \pm 2^{\circ} \mathrm{C}$ e umidade de $45 \%$.

Rev. Bras. PI. Med., Campinas, v.15, n.3, p.332-336, 2013. 


\section{Ação tópica}

No Laboratório de Entomologia da Embrapa Rondônia, os insetos foram borrifados com 0,25 $\mathrm{mL}$ das diluições do extrato em acetona a 100,0; 50,0; 25,0; 5,0 e $0,5 \mathrm{mg} \mathrm{mL}^{-1}$, utilizando-se acetona como controle. Os insetos foram colocados em placas de Petri de $9,0 \mathrm{~cm}$ de diâmetro, cobertas com papel filtro. Utilizou-se dez insetos por placa, em delineamento inteiramente casualizado com quatro repetições. Avaliou-se a mortalidade dos insetos em 48 horas em contato com o extrato. Na avaliação da mortalidade dos insetos, foram considerados vivos os insetos que moviam qualquer parte do corpo quando estimulados. Os dados foram submetidos à análise de variância e as médias comparadas pelo teste de Tukey.

\section{Exposição por superfície contaminada}

Foram utilizadas as mesmas soluções do bioensaio com aplicação tópica. Adicionou-se 1,0 $\mathrm{mL}$ das soluções mencionadas acima a placas de Petri de 9,0 cm de diâmetro, contendo papel de filtro. Após a evaporação da acetona, foram colocados dez insetos por placa, em quatro repetições, em delineamento inteiramente casualizado, avaliandose a mortalidade dos insetos durante as 48 horas seguintes. Os dados foram submetidos à análise de variância e as médias comparadas pelo teste de Tukey a $5 \%$ de probabilidade.

\section{Efeito de Repelência}

Frutos maduros de café sadio foram imersos em solução de extrato acetônico a $25,0 \mathrm{mg}$ $\mathrm{mL}^{-1}$, por um minuto, utilizando-se água destilada como controle. Placas de Petri de $9,0 \mathrm{~cm}$ de diâmetro foram demarcadas com uma linha central, sendo colocados três frutos de café tratados com solução de extrato de um lado e três frutos do grupo controle do outro lado. Na região central da placa, foram inseridos os insetos. Foi avaliado o número de insetos que se deslocaram para cada um dos lados de cada placa, durante os dez minutos seguintes. $O$ delineamento foi inteiramente casualizado com dez insetos por tratamento e dez repetições (placas). $\mathrm{O}$ índice de repelência foi calculado como indicado por Vilallobos \& Robledo (1998), R.I. = (C-T) / (C+T) $X 100$, onde $(C)$ é o número de insetos que se deslocavam para a área onde há dieta controle e (T) o número de insetos que se deslocavam para a área onde há dieta contendo frutos tratados. Os dados foram submetidos à análise de variância e as médias comparadas pelo teste de Tukey.

\section{RESULTADO E DISCUSSÃO}

O estudo fitoquímico do extrato acetônico das raízes de $P$. alatabaccum foi realizado no
Laboratório de pesquisa em química de produtos naturais (LPQPN) da Universidade Federal de Rondônia (UNIR), onde levou ao isolamento e identificação de três amidas, piperovatina, 8,9-dihidropiplartina e piplartina, e duas flavonas, 5,5',7-trimetoxi-3', 4'metilenodioxiflavona e $3^{\prime}, 4^{\prime}, 5,5^{\prime}, 7$-pentametoxiflavona. As três amidas e a flavona 5,5',7-trimetoxi-3',4'metilenodioxiflavona, já foram isoladas das folhas desta planta (Facundo et al., 2005). As estruturas de todos os compostos foram caracterizadas através dos espectros de Infravermelho, Massas e RMN ${ }^{1} \mathrm{H}$ e ${ }^{13} \mathrm{C}$ mono e bidimensional envolvendo comparação com dados relatados na literatura e comparação com amostras autênticas.

$\mathrm{Na}$ Tabela 1 estão apresentados os resultados do bioensaio em superfície contaminada, realizado com insetos de $H$. hampei, em relação a diferentes diluições do extrato de $P$. alatabaccum, no período de 48 horas. Os resultados mostram que o extrato utilizado foi eficiente na indução da mortalidade dos insetos e abre novas perspectivas quanto à utilização como inseticida no controle biológico de pragas. Logo na primeira hora do experimento, observou-se um efeito significativo do extrato, em todas as diluições testadas, sobre a mortalidade dos insetos. Na primeira hora de avaliação, a diluição de $100,0 \mathrm{mg} \mathrm{mL}^{-1}$ atingiu $100 \%$ de mortalidade. Esses dados vão de encontro aos experimentos de Santos et al. (2012), no qual ressalta que a velocidade em que o extrato provoca mortalidade pode ser de grande relevância, uma vez que, em condições de campo, o contato do inseto com o extrato pode ser relativamente rápido

Os resultados mostraram também que, nos demais períodos observados, a aplicação do extrato causou mortalidade significativa em relação ao controle. Mesmo na menor diluição utilizada ( 0,5 $\mathrm{mg} \mathrm{mL}^{-1}$ ) o extrato provocou $75 \%$ de mortalidade em 24 horas após o início do experimento. O efeito do extrato foi intenso até seis horas após a aplicação, estabilizando-se em seguida. Após este período, ocorreu apenas uma pequena variação, de $5 \%$, na diluição de $0,5 \mathrm{mg} \mathrm{mL}^{-1}$, mostrando que o efeito residual do extrato não é expressivo.

$\mathrm{Na}$ Tabela 2 estão apresentados os resultados do bioensaio utilizando aplicação tópica, realizado com insetos de $H$. hampei, em relação a diferentes diluições do extrato de $P$. alatabaccum, dentro de um período de 48 horas. Comparando-se as Tabelas 1 e 2, pode-se observar que, de forma geral, a aplicação tópica em superfície contaminada foi mais eficiente. A aplicação tópica também foi eficiente na promoção da mortalidade dos insetos, mas não atingiu $100 \%$, como ocorreu na aplicação em superfície contaminada. Provavelmente, isto está relacionado à capacidade de retenção do papel de

Rev. Bras. PI. Med., Campinas, v.15, n.3, p.332-336, 2013. 
TABELA 1. Efeito de diferentes diluições do extrato acetônico de raízes de $P$. alatabaccum sobre a mortalidade (\%) de insetos de $H$. hampei, em superfície contaminada, durante 48 horas.

\begin{tabular}{lllllllll}
\hline Diluições (mg mL-1) & \multicolumn{7}{c}{ Mortalidade (\%) } \\
\hline & \multicolumn{7}{c}{ Tempo (horas) } \\
\cline { 2 - 9 } & $\mathbf{1}$ & $\mathbf{2}$ & $\mathbf{3}$ & $\mathbf{4}$ & $\mathbf{5}$ & $\mathbf{6}$ & $\mathbf{2 4}$ & $\mathbf{4 8}$ \\
\cline { 2 - 9 } Controle & $0 \mathrm{f}$ & $0 \mathrm{e}$ & $0 \mathrm{f}$ & $0 \mathrm{e}$ & $0 \mathrm{~d}$ & $0 \mathrm{~d}$ & $5 \mathrm{~d}$ & $5 \mathrm{~d}$ \\
$0,5 \mathrm{mg} \mathrm{mL}^{-1}$ & $35 \mathrm{e}$ & $40 \mathrm{~d}$ & $40 \mathrm{e}$ & $55 \mathrm{~d}$ & $70 \mathrm{c}$ & $70 \mathrm{c}$ & $75 \mathrm{c}$ & $75 \mathrm{c}$ \\
$5,0 \mathrm{mg} \mathrm{mL}^{-1}$ & $45 \mathrm{~d}$ & $55 \mathrm{c}$ & $55 \mathrm{~d}$ & $65 \mathrm{c}$ & $80 \mathrm{~b}$ & $85 \mathrm{~b}$ & $85 \mathrm{~b}$ & $85 \mathrm{~b}$ \\
$25,0 \mathrm{mg} \mathrm{mL}^{-1}$ & $55 \mathrm{c}$ & $60 \mathrm{c}$ & $65 \mathrm{c}$ & $80 \mathrm{~b}$ & $85 \mathrm{~b}$ & $85 \mathrm{~b}$ & $85 \mathrm{~b}$ & $85 \mathrm{~b}$ \\
$50,0 \mathrm{mg} \mathrm{mL}^{-1}$ & $75 \mathrm{~b}$ & $80 \mathrm{~b}$ & $85 \mathrm{~b}$ & $100 \mathrm{a}$ & $100 \mathrm{a}$ & $100 \mathrm{a}$ & $100 \mathrm{a}$ & $100 \mathrm{a}$ \\
$100,0 \mathrm{mg} \mathrm{mL}^{-1}$ & $100 \mathrm{a}$ & $100 \mathrm{a}$ & $100 \mathrm{a}$ & $100 \mathrm{a}$ & $100 \mathrm{a}$ & $100 \mathrm{a}$ & $100 \mathrm{a}$ & $100 \mathrm{a}$ \\
\hline
\end{tabular}

*Letras diferentes indicam diferenças significativas, dentro de cada coluna, com $5 \%$ de probabilidade pelo teste de Tukey.

filtro, que proporcionou um contato mais prolongado dos insetos com as diluições do extrato. Porém, a aplicação tópica também foi eficiente, promovendo até $95 \%$ de mortalidade na diluição de $100,0 \mathrm{mg}$ $\mathrm{mL}^{-1}$. Da mesma forma que ocorreu no bioensaio em superfície contaminada, logo na primeira hora podese observar que houve efeito significativo do extrato, em todas as diluições testadas, em comparação com o controle do experimento.

Resultados semelhantes foram obtidos por Santos et al., (2010), avaliando a atividade do extrato das acetônico das folhas de Piper hispidum sobre $H$. hampei em superfície contaminada e ação tópica nas concentrações de 25, 5, 0.1, 0.02, 0.004, $0.0008 \mathrm{mg} \mathrm{mL}^{-1}$, obteve $100 \%$ de mortalidade com a maior concentração em 24 horas de experimento em superfície contaminada e $60 \%$ em aplicação tópica.

Estrela et al. (2006) ao avaliar o efeito da aplicação tópica dos óleos essenciais de $P$. aduncum e $P$. hispidinervum sobre a mortalidade de Sitophilus zeamais, em condições semelhantes a esse trabalho, observaram porcentagens significativas para todas as diluições avaliadas, atingindo mortalidade superior a $70 \%$.

Lima (2006) estudou a atividade inseticida do extrato etanólico de sementes de Pachira aquatica sobre $H$. hampei em aplicação tópica e superfície contaminada, verificando, em três horas, $33 \%$ de mortalidade, e $62 \%$ em 24 horas, nas diluições de 5 e $25,0 \mathrm{mg} \mathrm{mL}^{-1}$.

Portando, conforme os resultados observados neste trabalho, e de acordo com a literatura, a metodologia de aplicação mais eficiente para toxidade em insetos adultos, é a contaminação por superfície, onde, porém pode ser atribuída a novos testes em condições naturais de pré-campo e campo.

No bioensaio para repelência, em termos gerais, não foi obtido resultado positivo. Apenas foi possível identificar repelência no primeiro minuto, obtendo-se um índice de repelência igual a 103. De acordo com Villalobos \& Robledo (1998), podem ser consideradas repelentes substâncias cujo índice está acima de 50. Porém, após cinco e dez minutos, se obteve índices de 5,88 e 17,24, respectivamente, o que demonstra que o extrato não foi eficiente como

TABELA 2. Efeito de diferentes diluições do extrato acetônico de raízes de $P$. alatabaccum sobre a mortalidade de insetos de $H$. hampei, em aplicação tópica, durante 48 horas.

\begin{tabular}{lllllllll}
\hline Diluições $(\mathbf{m g ~ m L - 1 )}$ & \multicolumn{7}{c}{ Mortalidade (\%) } \\
\hline & \multicolumn{7}{c}{ Tempo (horas) } \\
\cline { 2 - 8 } & 1 & 2 & 3 & 4 & 5 & 6 & 24 & 48 \\
\cline { 2 - 8 } Controle & $0 \mathrm{e}$ & $0 \mathrm{e}$ & $0 \mathrm{e}$ & $5 \mathrm{e}$ & $5 \mathrm{e}$ & $10 \mathrm{f}$ & $15 \mathrm{e}$ & $15 \mathrm{~d}$ \\
$0,5 \mathrm{mg} \mathrm{mL}^{-1}$ & $10 \mathrm{~cd}$ & $15 \mathrm{c}$ & $15 \mathrm{c}$ & $25 \mathrm{bc}$ & $30 \mathrm{c}$ & $40 \mathrm{~cd}$ & $55 \mathrm{~d}$ & $80 \mathrm{c}$ \\
$5,0 \mathrm{mg} \mathrm{mL}^{-1}$ & $5 \mathrm{~d}$ & $5 \mathrm{~d}$ & $10 \mathrm{~d}$ & $20 \mathrm{~cd}$ & $30 \mathrm{c}$ & $35 \mathrm{de}$ & $55 \mathrm{~d}$ & $80 \mathrm{c}$ \\
$25,0 \mathrm{mg} \mathrm{mL}^{-1}$ & $5 \mathrm{~d}$ & $5 \mathrm{~d}$ & $10 \mathrm{~d}$ & $15 \mathrm{~d}$ & $15 \mathrm{~d}$ & $30 \mathrm{e}$ & $60 \mathrm{c}$ & $85 \mathrm{bc}$ \\
$50,0 \mathrm{mg} \mathrm{mL}^{-1}$ & $15 \mathrm{bc}$ & $40 \mathrm{a}$ & $50 \mathrm{a}$ & $60 \mathrm{a}$ & $70 \mathrm{a}$ & $80 \mathrm{~b}$ & $85 \mathrm{~b}$ & $90 \mathrm{ab}$ \\
$100,0 \mathrm{mg} \mathrm{mL}^{-1}$ & $25 \mathrm{a}$ & $30 \mathrm{~b}$ & $35 \mathrm{~b}$ & $60 \mathrm{a}$ & $65 \mathrm{~b}$ & $90 \mathrm{a}$ & $90 \mathrm{a}$ & $95 \mathrm{a}$ \\
\hline
\end{tabular}

*Letras diferentes indicam diferenças significativas, dentro de cada coluna, com $5 \%$ de probabilidade pelo teste de Tukey. 
repelente para $H$. hampei.

São muitos os estudos fitoquímicos realizados em varias espécies do gênero Piper, demonstrando a presença de uma diversidade de metabólitos secundários, incluindo alcalóides, amidas, propenilfenóis, ligninas, neoligninas, terpenos, esteróides, kawapirenos, chalconas, dihidrochalconas, flavonas e flavononas (DYER 2004). Dentre estes, destacamos as amidas como possível indicador da atividade inseticida do extrato acetônico das folhas de $P$. alatabaccum evidenciada neste trabalho. Entretanto, são necessários novos estudos com as substâncias isoladas, para comprovar esta ação, assim como experimentos envolvendo outros organismos, como os inimigos naturais de $\mathrm{H}$. hampei (Cephalonomia sp. e Beauveria bassiana, atualmente utilizados no controle biológico desta praga).

\section{AGRADECIMENTO}

Ao CNPq pela concessão de bolsa de Iniciação Científica a Renato A. Lima; de Produtividade em Pesquisa a Valdir A. Facundo, CENAUREM/UFC pela obtenção dos espectros de RMN ${ }^{1} \mathrm{H}$ e ${ }^{13} \mathrm{C}$ uni e bidimensional e ao Doutor Cid Ferreira do INPA pela identificação botânica das plantas.

\section{REFERÊNCIA}

BARREIRA, J.P. et al. Introducción de espécies de parasitoides africanos a México para el control biologico de la broca del cafe, Hypothenemus hampei (Ferrari) (Coleoptera: Scolytidae). Folia Entomologica Mexicana, v.79, n.3, p.245-7, 1990.

BUSTILLO, A.E. El manejo de cafetales y su relación con el control de la broca del cafe en Colombia. Colombia: Cenicafé, 2002. 40p.

CASTRO, P.R.C.; KLUGE, R.A.; PERES, L.E.P. Manual de fisiologia vegetal. São Paulo: Agronômica Ceres, 2005. 650p.

DYER, L. A., PALMER, A.D.N.Piper: A Model Genus for Studies of Pytochemistry, Ecology, and Evolution. Kluwer Academic Publisher/New York. 2004.

ESTRELA, J.L.V. et al. Toxicidade de óleos essenciais de Piper aduncum e Piper hispidinervum em Sitophilus zeamais. Pesquisa Agropecuária Brasileira, v.41, n.2, p.217-22, 2006.

FACUNDO, V.A.; MORAIS, S.M.; BRAZ-FILHO, R. Constituintes químicos de Ottonia corcovadensis Miq., da Floresta Amazônica: atribuições dos deslocamentos químicos dos átomos de hidrogênio e carbono. Química nova, v.27, n.1, p.79-83, 2004.

FACUNDO, V.A.; SILVEIRA, A.S.P.; MORAIS, S.M. Costituents of Piper alatabaccum Trel \& Yuncker (Piperaceae). Biochemical Systemy Ecology, v.33, p.753-6, 2005.

FAZOLIN, M; ESTRELA, J.L. V; CATANI, V.; LIMA, M.S.; ALECIO,M.R;. Toxidade do óleo de Piper aduncum L. a adultos de Cerotoma tingomarianos Bechyne (Coleoptera: Chrysomelidae). Neotropical Entomology. v. 34, n. 3, p. 485-489, 2005.

GUIMARÃES, R.J.; MENDES, A.N.G.; SOUZA, C.A. Estocagem e armazenagem: cafeicultura brasileira. Lavras: Universidade Federal de Lavras, 2002. 317p.

JARAMILLO, M.A.; MANOS, P.S. Phylogeny and patterns of floral diversity in the genus Piper (Piperaceae). American Journal of Botany, v.88, n.1, p.706-16, 2001.

LALE, N.E.S. A laboratory study of the comparative toxicity of products from three spices to the maize weevil. Postharvest Biology and Technology, v.2, n.1, p.61-4, 1992.

LIMA, D.K.S. Atividade inseticida e fungicida do extrato etanólico de Pachira aquatica Aubl. sobre Hypothenemus hampei Ferrari e Fusarium spp. 2006. 62p. Dissertação (Mestrado - Área de concentração em Biologia Experimental) - Departamento de Botânica, Universidade Federal de Rondônia, Porto Velho.

REIS, P.R.; SOUZA, J.C.; VENZON, M. Manejo ecológico das principais pragas do cafeeiro. Informe Agropecuário, v.214, n.23, p.83-99, 2002.

SANTOS, J.C.F. Cultura do café em Rondônia. Cafeicultura, v.4, n.1, p.22-4, 2002.

SANTOS, M.R.A.; SILVA, A.G.; LIMA, R.A.; LIMA, D.K.S.; SALLET, L.A.P.; TEIXEIRA, C.A.D.; POLLI, A.R.; FACUNDO, V.A. Atividade inseticida do extrato das folhas de Piper hispidum (Piperaceae)sobre a brocado-café (Hypothenemus hampei) Revista Brasil. Bot., v.33, n.2, p.319-324, 2010.

SILVA, W.C., RIBEIRO, J.D., SOUZA, H.E.M., CORREAA, R.S. Atividade inseticida de Piper aduncum L.(Piperaceae) sobre Aetalion SP. (Hemiptera: Aetalionidae), praga de importância econômica no Amazonas. Acta Amazonica. v. 37, n.2, p.293-298, 2007.

SOUZA, V.C.; LORENZI, H. Botânica sistemática: guia ilustrado para identificação das famílias de Fanerógamas nativas e exóticas no Brasil, baseado em APG II. 2.ed. Nova Odessa: Instituto Plantarum, 2008. 704p.

TRINDADE, F.T. T. et al. Evaluation of larvicidal activity of the methanolic extracts of Piper alatabaccum branches and $P$. tuberculatum leaves and compounds isolated against Anopheles darlingi. Rev. bras. farmacogn. [online]. ahead of print, pp. 0-0. Epub. Apr 03, 2012.

VILLALOBOS, M.J.P.; ROBLEDO, A. Screening for antiinsect activity in Mediterranean plants. Industrial Crops and Products, v.8, n.3, p.183-94, 1998. 